\title{
Structure Tensor of Colour Quaternion Image Representations for Invariant Feature Extraction
}

\author{
Jesús Angulo \\ CMM-Centre de Morphologie Mathématique, Mathématiques et Systèmes, MINES \\ Paristech; 35, rue Saint Honoré, 77305 Fontainebleau Cedex, France \\ jesus.angulo@ensmp.fr
}

\begin{abstract}
Colour image representation using real quaternions has shown to be very useful for linear and morphological colour filtering. This paper deals with the extension of first derivatives-based structure tensor for various quaternionic colour image representations. Classical corner and edge features are obtained from eigenvalues of the quaternionic colour structure tensors. We study the properties of invariance of the quaternion colour spatial derivatives and their robustness for feature extraction on practical examples.
\end{abstract}

\section{Introduction}

A colour point can be represented according to different geometric algebra structures. Real quaternions have been considered in last years to represent and to perform colour transformations by taking into account the $3 \mathrm{D}$ vector nature of colour triplets. Quaternion-based colour operations, such as colour Fourier transform, colour convolution and linear filters, have been studied mainly by 9 16 10 and by [7], and to build colour PCA by [18. Quaternion representations of normalized colours were used by [5] to construct edge colour detectors based on Prewitt edge detector. We have recently explored also the interest of colour quaternions for extending mathematical morphology to colour images [2].

Extraction of differential-based features such as edges, corners or salient points is a necessary low-level image processing task in many applications such as segmentation, tracking, object matching and object classification. These features are based on Gaussian-filtered combination of spatial derivatives [1] [3]. The most stable differential invariants involves lonely derivatives till order "one" [12]. Di Zenzo stated, in [8], that a simple summation of the derivatives ignores the correlation between the channels and proposed in his pioneer work a tensor representation of colour derivatives in order to compute the colour gradient by considering the colour image as a surface in $\mathbb{R}^{3}$. Later, Sochen et al. [19] considered a colour image as a two-dimensional manifold embedded in the five-dimensional non-Euclidean space whose coordinates are $(x, y, R, G, B) \in \mathbb{R}^{5}$ which is described by Beltrami colour metric tensor. Colour tensor based methods have been used in various colour feature detection algorithms [6] [15] [17 [13] [21.

Weijer et al. 22] proposed a framework to combine the differential-based features with the photometric invariance theory, in order to obtain colour

A. Trémeau, R. Schettini, and S. Tominaga (Eds.): CCIW 2009, LNCS 5646, pp. 91-100, 2009.

(C) Springer-Verlag Berlin Heidelberg 2009 
photometric invariant edges, corners, etc. We propose in this study a parallel framework combining the alternatives colour quaternion representations with the structure tensor. We focus also on the extraction of features such as edges and corners which present interesting colour invariance properties.

\section{Colour Quaternion Image Representations}

Colour Quaternion Representations. Let $\mathbf{c}=(r, g, b)$ be the triplet of the red, green and blue intensities for the pixel of a digital colour image. According to the previous works on the representation of colour by quaternions, we consider the gray-centered RGB colour-space [9]. In this space, the unit RGB cube is translated so that the coordinate origin $\hat{O}(0,0,0)$ represents mid-gray (middle point of the gray axis or half-way between black and white). In order to better exploit the power of quaternion algebra, we have recently proposed in [2] to represent each colour $\mathbf{c}$ by a full real quaternion $\mathbf{q}$ in its hypercomplex form, i.e., $\mathbf{c}=(r, g, b) \Rightarrow \mathbf{q}=\psi\left(\mathbf{c}, \mathbf{c}_{0}\right)+i \hat{r}+j \hat{g}+k \hat{b}$. where $\hat{\mathbf{c}}=(\widehat{r}, \widehat{g}, \widehat{b})=(r-1 / 2, g-$ $1 / 2, b-1 / 2)$. A quaternion has a real part or scalar part, $S(\mathbf{q})=\psi\left(\mathbf{c}, \mathbf{c}_{0}\right)$, and an imaginary part or vector part, $V(\mathbf{q})=\widehat{r} i+\widehat{g} j+\widehat{b} k$, such that the whole quaternion may be represented by the sum of its scalar and vector parts as $\mathbf{q}=S(\mathbf{q})+V(\mathbf{q})$. A quaternion with a zero real/scalar part is called a pure quaternion. The scalar component, $\psi\left(\mathbf{c}, \mathbf{c}_{0}\right)$, is a real value obtained from the current colour point and a colour of reference $\mathbf{c}_{0}=\left(r_{0}, g_{0}, b_{0}\right)$. The reference $\mathbf{c}_{0}$ can be for instance the white point $(1,1,1)$, but also any other colour which should impose a particular effect of the associated operator.

We have considered three possible definitions for the scalar part.

1) Saturation: $\psi\left(\mathbf{c}, \mathbf{c}_{0}\right)^{s a t}=s-1 / 2$, where $s$ is the saturation of the luminance/saturation/hue representation in norm $L_{1}$ [1].

2) Mass with respect to $\mathbf{c}_{0}: \psi\left(\mathbf{c}, \mathbf{c}_{0}\right)_{\lambda}^{\text {mass }}=\exp \left(-w_{E}\left\|\mathbf{c}-\mathbf{c}_{0}\right\|-w_{\angle} \arccos \right.$ $\left.\left(\frac{\mathbf{c} \cdot \mathbf{c}_{0}}{\|\mathbf{c}\|\left\|\mathbf{c}_{0}\right\|}\right)\right)$, where $w_{E}=(1 / \sqrt{2}) \lambda$ and $w_{\angle}=(2 \pi)^{-1}(1-\lambda)$, with $0 \leq \lambda \leq 1$.

3) Potential with respect to $\mathbf{c}_{0}$ and the nine significant colour points in the $R G B$ unit cube: $\psi\left(\mathbf{c}, \mathbf{c}_{0}\right)^{p o t}=\phi_{E}^{+}+\phi_{E}^{-}=\frac{\kappa^{+}}{4 \pi \epsilon_{0}\left\|\mathbf{c}-\mathbf{c}_{0}\right\|}+\sum_{n=-4}^{4} \frac{\kappa^{-}}{4 \pi \epsilon_{0}\left\|\mathbf{c}-\mathbf{c}^{n}\right\|}$, where the positive potential $\phi_{E}^{+}$represents the influence of a positive charge placed at the position of the reference colour $\mathbf{c}_{0}$ and the negative term $\phi_{E}^{-}$corresponds to the potential associated to nine negative charges in the significant colours of the RGB cube: (r,g,b,c,m,y,w,b and $\mathrm{mg}$ ), $\left\{\mathbf{c}^{n}\right\}_{n=-4}^{4}$.

More details on the properties of these scalar parts are given in 2 .

Any quaternion may be represented in polar form as $\mathbf{q}=\rho e^{\xi \theta}$, with $\rho=$ $\sqrt{a^{2}+b^{2}+c^{2}+d^{2}}, \xi=\frac{b i+c j+d k}{\sqrt{b^{2}+c^{2}+d^{2}}}$ and $\theta=\arctan \left(\frac{\sqrt{b^{2}+c^{2}+d^{2}}}{a}\right)$. In this polar formulation, $\rho=|\mathbf{q}|$ is the modulus of $\mathbf{q}$; $\xi$ is the pure unitary quaternion associated to $\mathbf{q}$ (by the normalisation, the quaternion representation of a colour discards distance information, but retains orientation information relative to mid-gray, which correspond in fact to the chromatic or hue-related information.), sometimes called eigenaxis; and $\theta$ is the angle, sometimes called eigenangle, 
between the real part and the 3D imaginary part. The eigenaxis of a colour quaternion, $\xi$, is independent of its scalar part. The imaginary term $b^{2}+c^{2}+$ $d^{2}=(r-1 / 2)^{2}+(g-1 / 2)^{2}+(b-1 / 2)^{2}$ is the norm of the colour vector in the centered cube and can be considered as a perceived energy of the colour (i.e., relative energy with respect to the mid-gray), being maximal for the eight significant colours associated to the cube corners. Note that the black and white have the same value as the six chromatic colours. The modulus $\rho$ is an additive combination of the imaginary part and the scalar part.

Using the product of quaternions, it is possible to describe vector decompositions. A full quaternion $\mathbf{q}$ may be decomposed about a pure unit quaternion $\mathbf{p}^{u}$ in its parallel/perpendicular form [9]: $\mathbf{q}=\mathbf{q}_{\perp}+\mathbf{q}_{\|}$, the parallel part of $\mathbf{q}$ according to $\mathbf{p}^{u}$, also called the projection part, is given by $\mathbf{q}_{\|}=S(\mathbf{q})+V_{\|}(\mathbf{q})$, and the perpendicular part, also named the rejection part, is obtained as $\mathbf{q}_{\perp}=V_{\perp}(\mathbf{q})$ where $V_{\|}(\mathbf{q})=\frac{1}{2}\left(V(\mathbf{q})-\mathbf{p}^{u} V(\mathbf{q}) \mathbf{p}^{u}\right)$ and $V_{\perp}(\mathbf{q})=\frac{1}{2}\left(V(\mathbf{q})+\mathbf{p}^{u} V(\mathbf{q}) \mathbf{p}^{u}\right)$.

In the case of colour quaternions, $\mathbf{p}^{u}$ corresponds to the pure unit quaternion associated to the reference colour $\mathbf{c}_{0}$, which is denoted $\mathbf{q}_{0}^{u}$. It should be remarked that the rejection part is a pure quaternion and that the value is independent of the scalar part of $\mathbf{q}$, but, of course, it depends on the reference colour used for the decomposition.

We can particularise the expression to obtain the following vectorial part and perpendicular part for the unit quaternion $\mathbf{c}_{0}=\left(r_{0}, r_{0}, r_{0}\right)\left(\Rightarrow \widehat{r}_{0, u}=1 / \sqrt{3}\right)$, which represents the decomposition along the grey axis:

$V_{\|}(\mathbf{q})=\frac{1}{3}[(\widehat{r}+\widehat{g}+\widehat{b}) i+(\widehat{r}+\widehat{g}+\widehat{b}) j+(\widehat{r}+\widehat{g}+\widehat{b}) k]$, and $V_{\perp}(\mathbf{q})=\frac{1}{3}[(2 \widehat{r}-\widehat{g}-\widehat{b}) i+(2 \widehat{g}-\widehat{r}-\widehat{b}) j+(2 \widehat{b}-\widehat{r}-\widehat{g}) k]$. We notice that these projection components correspond respectively to the luminance and the chromaticity terms [1]. Hence the colour image is decomposed into the intensity information along the grey axis (parallel part) and the chromatic information (perpendicular). Taking another example, for instance $\mathbf{c}_{0}=\left(r_{0}, r_{0} / 2,0\right)$ $\left(\Rightarrow \widehat{r}_{0, u}=\sqrt{\frac{4}{5}}\right)$ we have $V_{\|}(\mathbf{q})=\frac{4}{5}[(\widehat{r}+\widehat{g} / 2) i+(\widehat{r} / 2+\widehat{g} / 4) j]$ and $V_{\perp}(\mathbf{q})=$ $\frac{4}{5}(\widehat{r} / 4-\widehat{g} / 2) i+\frac{4}{5}(\widehat{g}-\widehat{r} / 2) j+\widehat{b} k$.

Quaternionic Colour Images. Let $\mathbf{f}(x, y)=\left(f_{R}(x, y), f_{G}(x, y), f_{B}(x, y)\right)$ be a colour image in the standard red, green and blue representation, i.e., a multivariate image of three vector components. Associating to each colour pixel the three alternative colour quaternion representations, and after fixing the scalar part $\psi_{\mathbf{c}_{0}}$ and consequently the reference colour $\mathbf{c}_{0}$, three quaternionic colour images can be defined. The hypercomplex quaternion colour image is denoted as $\mathbf{f}_{\text {hyper }}(x, y)=\left(f_{\psi_{\mathbf{c}_{0}}}(x, y), f_{i}(x, y), f_{j}(x, y), f_{k}(x, y)\right)$, where $f_{i}(x, y)=\hat{f}_{R}(x, y)$, $f_{j}(x, y)=\hat{f}_{G}(x, y)$ and $f_{k}(x, y)=\hat{f}_{G}(x, y)$. The polar quaternion colour image is given by the following 5 -variable function

$\mathbf{f}_{\text {polar }}(x, y)=\left(f_{\rho}(x, y), f_{\theta}(x, y), f_{\xi, i}(x, y), f_{\xi, j}(x, y), f_{\xi, k}(x, y)\right)$.

Finally, the parallel/perpendicular quaternion colour image is composed of two functions 


$$
\begin{aligned}
& \mathbf{f}_{\text {par } / \text { pen }}(x, y)=\left(\mathbf{f}_{\|}(x, y), \mathbf{f}_{\text {perp }}(x, y)\right)= \\
& \left(f_{\psi_{\mathbf{c}_{0}}}(x, y), f_{\|, i}(x, y), f_{\|, j}(x, y), f_{\|, k}(x, y), f_{\perp, i}(x, y), f_{\perp, j}(x, y), f_{\perp, k}(x, y)\right) .
\end{aligned}
$$

\section{2D Structure Tensor for Quaternionic Colour Images}

Spatial First-Derivatives and Structure Tensor. Given a multivariate image $\mathbf{i}(x, y)$, the $2 \mathrm{D}$ structure tensor is defined as [3]:

$$
\mathbf{G}(\mathbf{i})(x, y)=\omega_{\sigma} *\left(\nabla \mathbf{i}(x, y) \nabla \mathbf{i}(x, y)^{T}\right)=\left(\begin{array}{ll}
g_{x x}(x, y) & g_{x y}(x, y) \\
g_{x y}(x, y) & g_{y y}(x, y)
\end{array}\right)
$$

This $2 \times 2$ matrix represents the averaged dyadic product of the $2 \mathrm{D}$ spatial intensity gradient $\nabla \mathbf{i}(x, y)=\left(\frac{\partial \mathbf{i}(x, y)}{\partial x}, \frac{\partial \mathbf{i}(x, y)}{\partial y}\right)^{T}$, where $\omega_{\sigma}$ stands for a Gaussian smoothing with a standard deviation of $\sigma$. The elements of the tensor are invariant under rotation and translation of the spatial axes. This tensor does not be mistaken for the Hessian matrix which involves second-derivatives. In the case of a standard RGB colour image we have $\mathbf{G}(\mathbf{f})(x, y)$ with $g_{x x}(x, y)=$ $\omega_{\sigma} *\left(f_{R}^{x}\right)^{2}+\omega_{\sigma} *\left(f_{G}^{x}\right)^{2}+\omega_{\sigma} *\left(f_{B}^{x}\right)^{2}$ (idem. $g_{y y}(x, y)$ mutatis mutandis $f_{C}^{x}$ by $f_{C}^{y}$ ) and $g_{x y}(x, y)=\omega_{\sigma} *\left(f_{R}^{x} f_{R}^{y}\right)+\omega_{\sigma} *\left(f_{G}^{x} f_{G}^{y}\right)+\omega_{\sigma} *\left(f_{B}^{x} f_{B}^{y}\right)$, where to simplify the notation the spatial derivatives of colour component $C$ are $f_{C}^{x}=\frac{\partial f_{C}(x, y)}{\partial x}$ and $f_{C}^{y}=\frac{\partial f_{C}(x, y)}{\partial y}$.

Feature Detectors. The two real eigenvalues of the structure tensor $\mathbf{G}(x, y)$ at each point are given by $\lambda_{1,2}=\frac{1}{2}\left(g_{x x}+g_{y y} \pm \sqrt{\left(g_{x x}-g_{y y}\right)^{2}+\left(2 g_{x y}\right)^{2}}\right)$. These eigenvalues are correlated with the local image properties of edgeness and cornerness, i.e., $\lambda_{1}>>0$ and $\lambda_{2} \approx 0$ and $\lambda_{1} \approx \lambda_{2}$ respectively. More precisely, based on the spatial functions $\lambda_{1}(x, y)$ and $\lambda_{2}(x, y)$ several edge and corner feature indicator functions have been proposed in the literature.

The classical Harris and Stephen corner detector [11] works on the Gaussian curvature of the surface, and this quantity is corrected by the square average of the principal curvature: $H(\mathbf{i})(x, y)=\operatorname{det}(\mathbf{G}(x, y))-k \operatorname{trace}(\mathbf{G}(x, y))^{2}=$ $\lambda_{1}(x, y) \lambda_{2}(x, y)-k\left(\lambda_{1}(x, y)+\lambda_{2}(x, y)\right)^{2}$, with typically $k=0.04$. Instead of this original formulation, we propose to use for corner detection the modification proposed by Noble [14] which leads to better results without needing the parameter $k$ :

$$
N(\mathbf{i})(x, y)=\frac{\lambda_{1}(x, y) \lambda_{2}(x, y)}{\lambda_{1}(x, y)+\lambda_{2}(x, y)} .
$$

Most of previous works consider the magnitude of $\lambda_{1}(x, y)$ for edge detection. We prefer here the sounder theoretical approach introduced by Sochen et al. [19] based on the determinant of the Beltrami colour metric tensor $\widehat{\mathbf{G}}$. The colour metric tensor can be reformulated as function of the $2 \mathrm{D}$ structure tensor 4 : $\widehat{\mathbf{G}}(\mathbf{i})(x, y)=\mathbf{I}_{2}+\mathbf{G}(\mathbf{i})(x, y)$, where $\mathbf{I}_{2}$ is the identity matrix. The Beltrami colour edge can now be defined as 


$$
\begin{array}{r}
B(\mathbf{i})(x, y)=\operatorname{det}(\widehat{\mathbf{G}}(x, y))=1+\operatorname{trace}(\mathbf{G}(x, y))+\operatorname{det}(\mathbf{G}(x, y)) \\
=1+\left(\lambda_{1}(x, y)+\lambda_{2}(x, y)\right)+\lambda_{1}(x, y) \lambda_{2}(x, y) .
\end{array}
$$

\section{Adaptation to Quaternionic Representations: Quaternionic Image} Derivatives. The various quaternionic image representations involve different structure tensor values. Here we formulate the quaternionic image derivatives in terms of the RGB spatial derivatives, i.e., $f_{R}^{x}, f_{G}^{x}, f_{B}^{x}$ for $x$-derivative. The idea of this calculation is basically to apply the derivative Chain Rule. To be more precise, let $r=r(x, y), g=g(x, y)$ and $b=b(x, y)$ have first-order partial derivatives at the point $(x, y)$ and suppose that the multivariate function $\mathbf{i}=\mathbf{i}(r, g, b)$ $=(r(x, y), g(x, y), b(x, y))$ is differentiable in $r, g$ and $b$. The first-order partial derivative at point $(x, y)$ is given by $\frac{\partial \mathbf{i}}{\partial x}=\frac{\partial \mathbf{i}}{\partial r} \frac{\partial r}{\partial x}+\frac{\partial \mathbf{i}}{\partial g} \frac{\partial g}{\partial x}+\frac{\partial \mathbf{i}}{\partial b} \frac{\partial b}{\partial x}$, and idem. for $\frac{\partial \mathbf{i}}{\partial y}$.

Let us start by the scalar part. For the saturation it is obtained that,

$$
f_{\psi_{s a t, \mathbf{c}_{0}}^{x}}^{x}=\left\{\begin{array}{l}
\frac{3}{2} f_{\text {max }}^{x}-\frac{1}{2} f_{R}^{x}-\frac{1}{2} f_{G}^{x}-\frac{1}{2} f_{B}^{x} \text { if } \quad f_{m} \geq f_{m e d} \\
\frac{1}{2} f_{R}^{x}+\frac{1}{2} f_{G}^{x}+\frac{1}{2} f_{B}^{x}-\frac{3}{2} f_{\text {min }}^{x} \text { if } \quad f_{m} \leq f_{m e d},
\end{array}\right.
$$

and identically for $f_{\psi_{s a t, \mathbf{c}_{0}}}^{y}$. The term $\left(f_{\psi_{s a t, \mathbf{c}_{0}}^{x}}^{x}\right)$ is quasi-invariant to specular changes [21], and consequently by this way $\mathbf{f}_{h y p e r}^{x}$ decorrelates the derivative with respect to specular variations; note that $\left(f_{i}^{x}, f_{j}^{x}, f_{k}^{x}\right)$ is the specular variant part.

In the case of the mass with respect to $\mathbf{c}_{0}$, for the sake of simplicity we fix $\lambda=1$, and we have:

$f_{\psi_{\text {mass }, \mathbf{c}_{0}}^{x}}^{x}=\Theta\left(\mathbf{c}_{0}\right)\left[\left(f_{R}-r_{0}\right) f_{R}^{x}+\left(f_{G}-g_{0}\right) f_{G}^{x}+\left(f_{B}-b_{0}\right) f_{B}^{x}\right]$, where $\Theta\left(\mathbf{c}_{0}\right)=-w_{E}\left(\Delta \mathbf{c}_{0}\right)^{-1 / 2} \exp \left(-w_{E}\left(\Delta \mathbf{c}_{0}\right)^{1 / 2}\right)$ with $\Delta \mathbf{c}_{0}=\left(f_{R}-r_{0}\right)^{2}+$ $\left(f_{G}-g_{0}\right)^{2}+\left(f_{B}-b_{0}\right)^{2}$. In the case of the potential function as scalar part, the derivative involves quite similar terms:

$f_{\psi_{\text {pot }, \mathbf{c}_{0}}}^{x}=-9 Q\left(\Delta \mathbf{c}_{0}\right)^{-3 / 2}\left[\left(f_{R}-r_{0}\right) f_{R}^{x}+\left(f_{G}-g_{0}\right) f_{G}^{x}+\left(f_{B}-b_{0}\right) f_{B}^{x}\right]$

$+\sum_{n=-4}^{4} Q\left(\Delta \mathbf{c}_{n}\right)^{-3 / 2}\left[\left(f_{R}-r_{n}\right) f_{R}^{x}+\left(f_{G}-g_{n}\right) f_{G}^{x}+\left(f_{B}-b_{n}\right) f_{B}^{x}\right]$.

We observe that both $f_{\psi_{\text {mass }, \mathbf{c}_{0}}}^{x}$ and $f_{\psi_{\text {pot }, \mathbf{c}_{0}}}^{x}$ weight each colour derivative with the corresponding distance term to the reference colour. Using these terms in $\mathbf{f}_{\text {hyper }}^{x}$ we are able to introduce a particular effect with respect the reference colour $\mathbf{c}_{0}$.

The derivative in the polar representation $\mathbf{f}_{\text {polar }}^{x}$ has, on the one hand, the three intensity normalised derivatives from $\xi$, i.e., $f_{\xi, i}^{x}=f_{R}^{x} / \sqrt{\mu}, f_{\xi, j}^{x}=f_{G}^{x} / \sqrt{\mu}$, $f_{\xi, k}^{x}=f_{B}^{x} / \sqrt{\mu}$, where $\mu=f_{i}^{2}+f_{j}^{2}+f_{k}^{2}$. For the modulus and the eigenangle, it is achieved respectively $f_{\rho}^{x}=\frac{f_{R} f_{R}^{x}+f_{G} f_{G}^{x}+f_{B} f_{B}^{x}}{\sqrt{\psi_{\mathbf{c}_{0}}+\mu}}+\frac{\psi_{\mathbf{c}_{0}} \psi_{\mathbf{c}_{0}}^{x}}{\sqrt{\psi_{\mathbf{c}_{0}}+\mu}}$ and $f_{\theta}^{x}=\frac{\psi_{\mathbf{c}_{0}}}{\sqrt{\mu}\left(\psi_{\mathbf{c}_{0}}^{2}+\mu\right)}\left(f_{R} f_{R}^{x}+f_{G} f_{G}^{x}+f_{B} f_{B}^{x}\right)-\frac{\sqrt{\mu}}{\left(\psi_{\mathbf{c}_{0}}^{2}+\mu\right)} \psi_{\mathbf{c}_{0}}^{x}$. These last two expressions depend particularly on the derivative of the scalar part. But in any case, 
quite complex factors weight the values of each colour derivative; and consequently their precise analytical analysis is not easy, even if their practical interest can be explored from empirical examples.

As shown in Section 2, the parallel/perpendicular quaternionic variables depend strongly on the reference $\mathbf{c}_{0}$. Choosing the significant point $\mathbf{c}_{0}=(1,1,1)$, the derivatives of the parallel quaternion image are:

$\mathbf{f}_{\|}^{x}=\left(\frac{f_{R}^{x}+f_{G}^{x}+f_{B}^{x}}{3}, \frac{f_{R}^{x}+f_{G}^{x}+f_{B}^{x}}{3}, \frac{f_{R}^{x}+f_{G}^{x}+f_{B}^{x}}{3}\right)$ and

$\mathbf{f}_{\perp}^{x}=\left(\frac{2}{3} f_{R}^{x}-\frac{1}{3} f_{G}^{x}-\frac{1}{3} f_{B}^{x}, \frac{2}{3} f_{G}^{x}-\frac{1}{3} f_{R}^{x}-\frac{1}{3} f_{B}^{x}, \frac{2}{3} f_{B}^{x}-\frac{1}{3} f_{R}^{x}-\frac{1}{3} f_{G}^{x}\right)$. Hence, this decomposition with $\mathbf{c}_{0}=(1,1,1)$ decorrelates the derivative information into an intensity variant part and the shadow-shading-specular quasi-invariant part. In addition, the choice of other references $\mathbf{c}_{0}$ leads to other interesting decorrelations in this framework. For instance, the simplest $\mathbf{c}_{0}=(1,0,0)$ produces $\mathbf{f}_{\|}^{x}=\left(f_{R}^{x}, 0,0\right)$ and $\mathbf{f}_{\perp}^{x}=\left(0, f_{G}^{x}, f_{B}^{x}\right)$, that is a decomposition along the red derivative and the orthogonal green/blue derivatives. In a similar way, for the colour $\mathbf{c}_{0}=(1,1 / 2,0)$ it is obtained $\mathbf{f}_{\|}^{x}=\left(\frac{4}{5} f_{R}^{x}+\frac{2}{5} f_{G}^{x}, \frac{2}{5} f_{R}^{x}+\frac{1}{5} f_{G}^{x}, 0\right)$ and $\mathbf{f}_{\perp}^{x}=\left(\frac{1}{5} f_{R}^{x}-\frac{2}{5} f_{G}^{x}, \frac{4}{5} f_{G}^{x}-\frac{2}{5} f_{R}^{x}, f_{B}^{x}\right)$. For many practical situations, it is better to take advantage of the decomposition and to use separately $\mathbf{f}_{\|}^{x}$ and $\mathbf{f}_{\perp}^{x}$. Note also that the global derivative of function $\mathbf{f}_{\text {par/pen }}^{x}$ includes also the term of the scalar part $f_{\psi_{\mathbf{c}_{0}}}^{x}$ associated to the parallel quaternion.

\section{Application to Invariant Feature Detection}

We propose now to extract significant features from the corner energy image $N(\mathbf{i})(x, y)$ and the edge energy image $B(\mathbf{i})(x, y)$. These quite simple algorithms are based on morphological tools.

Colour Corner Extraction. The corner extraction from image $N(\mathbf{i})(x, y)$ requires two classical steps.

1) The local maxima within a ball of radius $R$ are obtained by finding the invariant points to a gray level dilation, i.e., $M(x, y)=1$ if $\delta_{R}(N(\mathbf{i}))(x, y)=$ $N(\mathbf{i})(x, y)$, otherwise $M(x, y)=0$. For all our examples, $R=3 \times 3$ pixels. This binary mask is used to recover the initial intensities at the maxima points: $m(x, y)=N(\mathbf{i})(x, y) \times M(x, y)$ (where $\times$ is the pointwise multiplication).

2) Thresholding the function $m(x, y)$ at value $u_{\max }$ to keep only the most significant maxima: $\hat{M}(x, y)=1$ iff $\hat{m}(x, y)>u_{\max }$, where the parameter is defined as a percentage of the maximal intensity of $N(\mathbf{i}), u_{\max }=\left(\alpha_{\max } / 100\right) \max (N(\mathbf{i})$ $(x, y))$. For all our examples, $\alpha_{\max }=4 \%$.

Colour Geometry Sketch. The colour geometry sketch, more original, can be interpreted as a rough definition of the main colour image edges. The idea is to have a representation of the distribution of the edges according to their colour and geometry (size, orientation, etc.) without defining the regions. This simple descriptor can be useful for tracking, registering, etc. The needed steps are:

1) Intensity transformation of image $N(\mathbf{i})(x, y)$ using a $\gamma$-correction function 


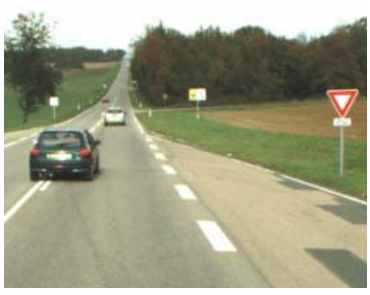

$\mathbf{f}(x, y)$

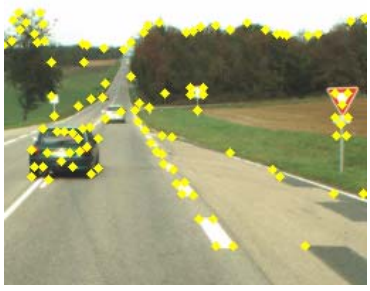

$N\left(\mathbf{f}_{\text {hyper }}\right)(x, y)$

$f_{\psi_{\text {sat }}, \mathbf{c}_{0}=(1,1,1)}$

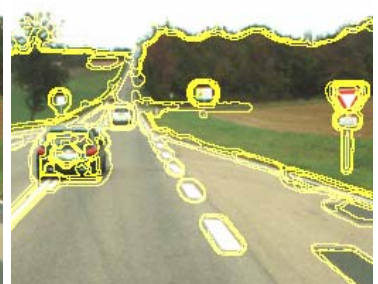

$B\left(\mathbf{f}_{\text {hyper }}\right)(x, y)$

$f_{\psi_{\text {sat }}, \mathbf{c}_{0}=(1,1,1)}$

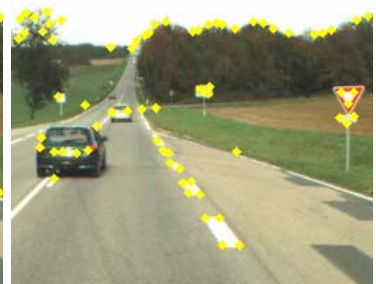

$N\left(\mathbf{f}_{\text {hyper }}\right)(x, y)$

$f_{\psi_{\text {mass }}, \mathbf{c}_{0}=(1,0,0)}$

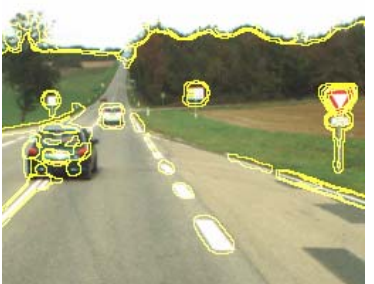

$B\left(\mathbf{f}_{\|}\right)(x, y)$

$f_{\psi_{\text {mass }}, \mathbf{c}_{0}=(1,0,0)}$

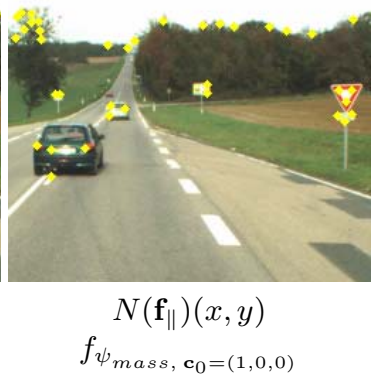

Fig. 1. Comparison with the image "Road" (from [20]) of colour geometry sketch for two Beltrami quaternionic colour edge energy functions $B(\mathbf{i})(x, y)$ and of colour corners for three Noble quaternionic colour corner energy functions $N(\mathbf{i})(x, y)$

followed by adjustment of dynamics in a discrete interval $\left[t_{\min }, t_{\max }\right]: e(x, y)=$ $(N(\mathbf{i})(x, y))^{\gamma}$ and $\hat{e}(x, y)=t_{\min }+\left\lceil\frac{e(x, y)-\min (e(x, y))}{\max (e(x, y))-\min (e(x, y))}\right\rceil t_{\max }$. For all our examples $\gamma=2$ and $\left[t_{\text {min }}, t_{\max }\right]=[0,255]$.

2) Extraction of the contours of the flat-zones of image $\hat{e}$ having a minimal

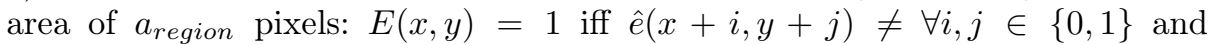
$\operatorname{Area}(R(x, y))>a_{\text {region }}$, where $R(x, y)$ is the connected component associated to point $(x, y)$. In practice, this step is implemented using a region growing procedure to remove the regions of size $\leq a_{\text {region }}$. For all our examples $a_{\text {region }}=50$ pixels.

3) Contour filtering using the supremum of orientated linear openings of size $l_{\text {min }}$ : $S(x, y)=\bigvee_{\beta} \gamma_{l_{m i n}, \beta}^{L}$, where the used orientations are $\beta=\left\{0^{\circ}, 45^{\circ}, 60^{\circ}, 90^{\circ}, 120^{\circ}\right.$, $\left.135^{\circ}\right\}$. The aim is to regularize the contours and to keep only those of length upper than $l_{\text {min }}$ according to the 6 main directions of the discrete grid. In fact, the preserved contour segments are then valued with the original colours: $s(x, y)=S(x, y) \times \mathbf{f}(x, y)$.

For all the examples of this paper, the Gaussian derivatives are computed with $\sigma=2$. In Fig. 1 is given an example with a road image from ROMA 

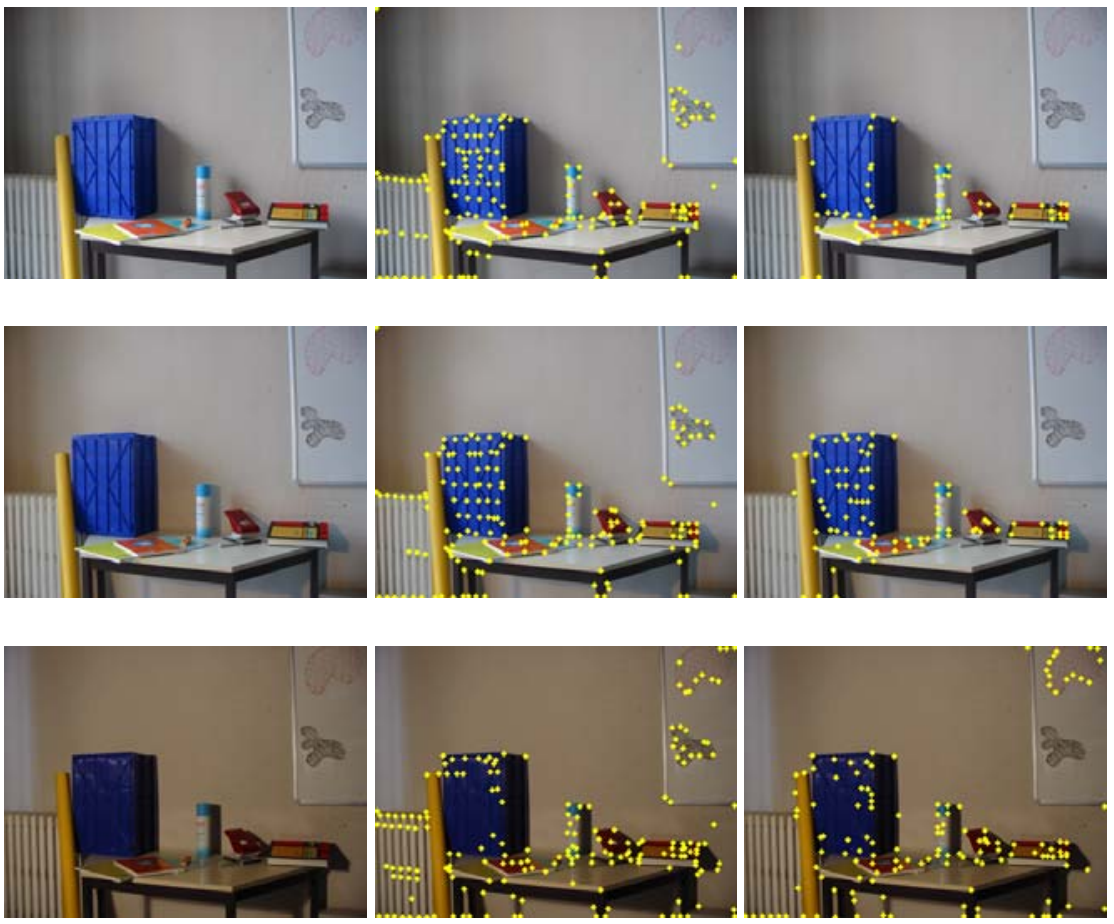

$\mathbf{f}(x, y)$

$$
\begin{gathered}
N\left(\mathbf{f}_{\text {par } / \text { pen }}\right)(x, y) \\
f_{\psi_{\text {mass }, \mathbf{c}_{0}}=(1,1,1)}
\end{gathered}
$$

$$
\begin{gathered}
N\left(\mathbf{f}_{\perp}\right)(x, y) \\
f_{\psi_{\text {mass }}, \mathbf{c}_{0}=(1,1,1)}
\end{gathered}
$$

Fig. 2. Invariance of colour corners extraction using two Noble quaternionic colour corner energy functions $N(\mathbf{i})(x, y)$. The three images were acquired from the same scene under different lighting conditions (natural light, fluorescence tube, tungsten bulb).

database [20]. The aim of this comparative example is to illustrate the colour geometry sketch and the colour corner extraction, and in particular to show how the effect associated to particular reference colour can be imposed. As we can observe, the hypercomplex representation with $\mathbf{c}_{0}=(1,1,1)$ extract the main contours and corners independently of their colour. If we focus on the red features by fixing $\mathbf{c}_{0}=(1,0,0)$, the parallel part representation focus more specifically on the target colour structure, although of course, other very significant unsaturated structures are also detected. The illumination invariance of colour corners extraction is illustrated in Fig. 2, where the three images were acquired from the same scene under different lighting conditions (natural light, fluorescence tube, tungsten bulb). We verify in particular, as expected in theory, that the chromatic information represented by the perpendicular part to $\mathbf{c}_{0}=(1,1,1)$ is more robust than the intensity information (i.e., the parallel part). Using two images differing from the viewpoint and orientation of lighting, and including highlights, we evaluate in Fig. 3 the invariance of colour geometry sketch using two Beltrami 

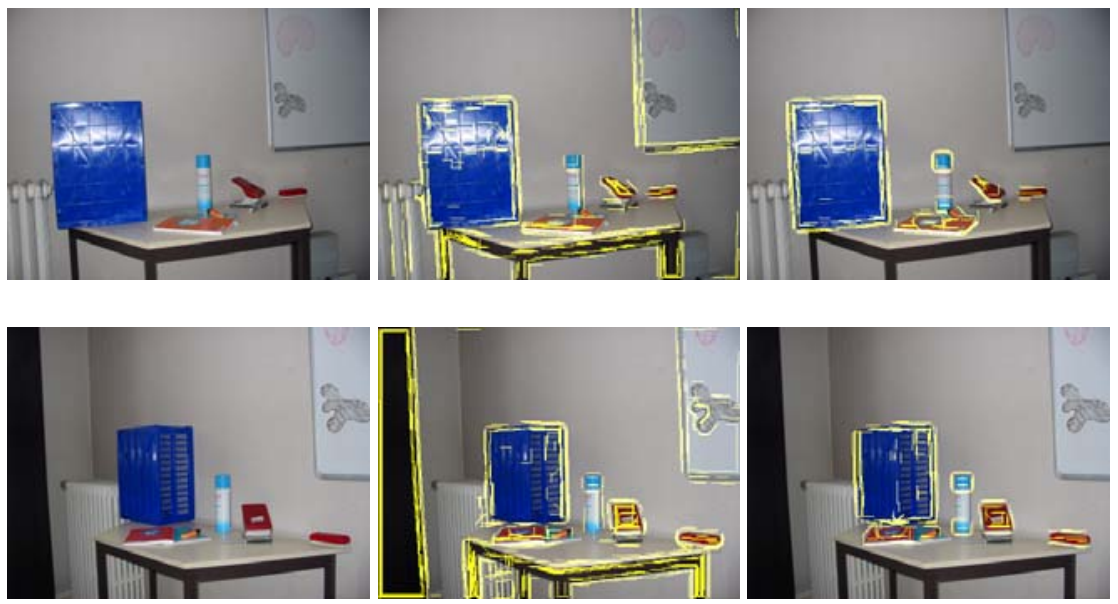

$\mathbf{f}(x, y)$

$$
\begin{gathered}
B\left(\mathbf{f}_{\text {hyper }}\right)(x, y) \\
f_{\psi_{\text {mass }, \mathbf{c}_{0}}=(1,1,1)}
\end{gathered}
$$

$$
\begin{gathered}
B\left(\mathbf{f}_{\perp}\right)(x, y) \\
f_{\psi_{\text {mass }, \mathbf{c}_{0}}=(1,1,1)}
\end{gathered}
$$

Fig. 3. Invariance of colour geometry sketch using two Beltrami quaternionic colour edge energy functions $B(\mathbf{i})(x, y)$. The two images differing from the viewpoint and orientation of lighting.

quaternionic colour edge energy function. We observe again that the chromatic information involves more robust and invariant features than the global colour information provided in this example by the hypercomplex representation.

\section{Conclusion}

In this paper, we proposed the extension of first derivatives-based structure tensor for various quaternionic colour image representations. From the quaternionic colour structure tensor, classical corner and edge features have been derived, obtaining in particular colour corners and colour geometry sketch. Experiments show that the colour quaternion-based features are more flexible and powerful than the RGB counterparts; in particular, it is possible to focus on features associated to a particular colour and their invariance properties lead to robust extraction results.

\section{References}

1. Angulo, J., Serra, J.: Modelling and Segmentation of Colour Images in Polar Representations. Image and Vision Computing 25(4), 475-495 (2007)

2. Angulo, J.: Quaternion colour representations and derived total orderings for morphological operators. In: CGIV 2008, pp. 417-422 (2008) 
3. Bigun, J., Granlund, G., Wiklund, J.: Multidimensional orientation estimation with applications to texture analysis and opitcal flow. IEEE Trans. Patt. Anal. and Mach. Intell. 13(8), 775-790 (1991)

4. Bunyak, F., Palaniappan, K., Nath, S.K., Seetharaman, G.: Flux tensor constrained geodesic active contours with sensor fusion and persistent object tracking. Journal of Multimedia 2(4), 20-33 (2007)

5. Cai, C., Mitra, S.K.: A normalized color difference edge detector based on quaternion representation. In: ICIP 2000 (2000)

6. Cumani, A.: Edge detection in multispectral images. CVGIP: Graphical Models and Image Processing 53(1) (1991)

7. Denis, P., Carré, P., Fernandez-Maloigne, C.: Spatial and spectral quaternionic approaches for colour images. Computer Vision and Image Understanding 107(23), 74-87 (2007)

8. Di Zenzo, S.: A note on the gradient of a multi-image. Computer Vision, Graphics, and Image Processing 33(1), 116-125 (1986)

9. Ell, T.A., Sangwine, S.J.: Hypercomplex Wiener-Khintchine theorem with application to color image correlation. In: IEEE ICIP 2000, vol. II, pp. 792-795 (2000)

10. Ell, T.A., Sangwine, S.J.: Hypercomplex Fourier transform of color images. IEEE Transactions on Image Processing 16(1), 22-35 (2007)

11. Harris, C., Stephens, M.: A combined corner and edge detector. In: Proc. 4th Alvey Vision Conf., vol. 15, pp. 147-151 (1988)

12. Montesinos, P., Gouet, V., Deriche, R.: Differential invariants for color images. In: IAPR International Conference on Pattern Recognition (ICPR 1998), pp. 838-840 (1998)

13. Naik, S.K., Murthy, C.A.: Standardization of edge magnitude in color images. IEEE Trans. on Image Processing 15(9), 2588-2595 (2006)

14. Noble, J.A.: Finding corners. Image and Vision Computing 6(2), 121-128 (1988)

15. Ruzon, M.A., Tomasi, C.: Edge, junction, and corner detection using color distributions. IEEE Trans. Patt. Anal. and Mach. Intell. 23(11), 1281-1295 (2001)

16. Sangwine, S.J., Ell, T.A.: Mathematical approaches to linear vector filtering of colour images. In: CGIV 2002, pp. 348-351 (2002)

17. Schmid, C., Mohr, R., Bauckhage, C.: Evaluation of interest point detectors. International Journal of Computer Vision 37(2), 151-172 (2000)

18. Shi, L., Funt, B.: Quaternion color texture segmentation. Computer Vision and Image Understanding 107(1-2), 88-96 (2007)

19. Sochen, N., Kimmel, R., Malladi, R.: A general framework for low level vision. IEEE Transactions on Image Processing 7(3), 310-318 (1998)

20. Veit, T., Tarel, J.-P., Nicolle, P., Charbonnier, P.: Evaluation of Road Marking Feature Extraction. In: Proceedings of 11th IEEE Conference on Intelligent Transportation Systems (ITSC 2008), Beijing, China, October 12-15 (2008)

21. van de Weijer, J., Gevers, T., Geusebroek, J.M.: Edge and corner detection by photometric quasi-invariants. IEEE Trans. Patt. Anal. and Mach. Intell. 27(4) (2005)

22. van de Weijer, J., Gevers, T., Smeulders, A.W.M.: Robust Photometric Invariant Features From the Colour Tensor. IEEE Trans. on Image Processing 15(1), 118-127 (2006) 